\title{
O PERFIL dAS REAdMISSÕES DE IDOSOS NUM HOSPITAL GERAL DE MARÍLIA/SP
}

Márcia Regina Martins Alvarenga ${ }^{1}$ Maria Manuela Rino Mendes ${ }^{2}$

Alvarenga MRM, Mendes MMR. O perfil das readmissões de idosos num hospital geral de Marília/SP. Rev Latino-am Enfermagem 2003 maio-junho; 11(3):305-11.

Estudo descritivo sobre readmissões de idosos em hospital geral, no município de Marília-SP, em 1996/1997. Objetivos: Caracterizar o perfil das readmissões, quanto às variáveis sexo, idade, estado civil, naturalidade, profissão, diagnósticos principal e secundário, tempo de permanência hospitalar, tipo de saída, fonte de financiamento, tipo de procedimento e intervalo de tempo entre as admissões; analisar registros da enfermagem, nos prontuários, sobre as condições à admissão e alta. Construído banco de dados sobre admissões, adotando o "software" Epi-Info 6. Pesquisados 262 idosos (53,8\% mulheres) que apresentaram 502 readmissões; 32,8\% destes foram readmitidos uma vez e 55,9\% retornaram à instituição em menos de 60 dias. Média de idade 73,2 anos e de permanência hospitalar 5,49 dias. Predominaram as doenças crônicas respiratórias e $55,8 \%$ das readmissões apresentaram diagnóstico secundário. Análise do conteúdo dos registros permitiu identificar a rotina da enfermagem, revelando apenas alguns aspectos da condição dos idosos à recepção e saída.

DESCRITORES: readmissão do paciente, idoso, enfermagem

\section{THE PROFILE OF ELDERLY PATIENTS' READMISSIONS IN A GENERAL HOSPITAL LOCATED IN MARÍLIA/SP}

This is a descriptive study about the readmissions of elderly patients in a general hospital located in the city of Marilia, São Paulo, Brazil, in 1996 and 1997. Objectives: characterizing the readmission profile with respect to the following variables: gender, age, marital status, origin, profession, main and secondary diagnoses, time of hospitalization, type of discharge, financing source, type of procedure and interval between hospitalizations; analyzing the nursing notes in the patients' records about health conditions at the time of admission and discharge. A database about admissions was compiled by using the Epi-Info 6 software. The research population consisted of two hundred sixty-two elderly patients (53.8\% of whom were female), who presented 502 readmissions; $32.8 \%$ of these occurred once and $55.9 \%$ took place in less than 60 days. The patients' mean age was 73.2 years and the average time of hospitalization was 5.49 days. Chronic respiratory diseases predominated and $55.8 \%$ of the readmitted patients presented a secondary diagnosis. The content analysis of the notes identified the nursing routine and revealed only some of the aspects concerning the elderly persons' conditions at the time of admission and discharge.

DESCRIPTORS: patient readmission, elderly, nursing

\section{EL PERFIL DE LOS REINGRESOS DE ANCIANOS EN UN HOSPITAL GENERAL DEL MUNICIPIO DE MARÍLIA, SP}

Estudio descriptivo sobre el reingreso del anciano en un hospital general en el municipio de Marília-SP, en 1996/1997. Objetivos: caracterizar el perfil de los reingresos en relación con las variables sexo, edad, estado civil, naturalidad, profesión, diagnósticos principal y secundario, tiempo de permanencia hospitalaria, tipo de salida, fuente de financiación, tipo de procedimiento e intervalo de tiempo entre los reingresos; analizar registros de enfermería en las historias clínicas de los ancianos, sobre sus condiciones de admisión y alta. Se construyó un banco de datos sobre admisiones, adoptando el "software" Epi-Info 6. Se investigaron 262 ancianos (53,8\% mujeres) que presentaron 502 reingresos; 32,8\% de estos reingresaron una vez y 55,9\% retornaron a la institución en menos de 60 días. El promedio de edad fue de 73,2 años y la estancia hospitalaria de 5,49 días. Predominaron las enfermedades crónicas del sistema respiratorio y 55,8\% de los reingresos presentaron diagnóstico secundario. El análisis de contenido de las notas de enfermería identificó rutina en la práctica de enfermería revelando solamente algunos aspectos de la situación de los ancianos a la recepción y la salida.

DESCRIPTORES: readmisión del paciente, anciano, enfermería

\footnotetext{
${ }^{1}$ Professor da Universidade Estadual de Mato Grosso do Sul, e-mail: marciarma@uems.br; ${ }^{2}$ Professor Doutor da Escola de Enfermagem da Universidade de São Paulo, Centro Colaborador da OMS para o desenvolvimento da pesquisa em enfermagem
} 
INTRODUÇÃO

A população idosa é a que mais adoece e, freqüentemente, acometida por uma ou mais doenças. A morbidade múltipla entre idosos está associada às doenças crônicas, bem como às condições precárias de vida ligadas à baixa renda, que requerem medidas de diversas naturezas, quer na área social, quer na saúde, implicando em políticas de formação profissional e destinação de recursos, instrumentos tecnológicos que envolvem altos custos. A complexidade da condição de vida dos idosos, associada às demais questões da sociedade brasileira, tem gerado novas políticas (recentes) que ainda não provocaram as mudanças esperadas.

No município de São Carlos-SP, em 1996, caracterizou-se a morbidade hospitalar de 4125 admissões de idosos (60 anos ou mais) residentes na cidade, que representaram $18,4 \%$ do total geral de admissões. Esses eventos foram gerados por 2829 idosos, sendo que com 510 (18\%) ocorreu uma vez, 137 (4,8\%) duas vezes e 77 $(2,7 \%)$ três. O maior número de admissões por idoso foi doze e a média de permanência hospitalar foi 6,1 dias $^{(1)}$.

No Brasil, em 1997, as taxas de admissão hospitalar e o tempo médio de ocupação do leito por idosos são mais elevados quando comparados aos outros grupos etários, exceto às decorrentes do parto e suas complicações. A falta de serviços domiciliares e ambulatoriais adequados faz com que o primeiro atendimento à saúde aconteça em estágio avançado do quadro e no hospital, aumentando os custos e o número das admissões e diminuindo a possibilidade de prognósticos mais favoráveis ${ }^{(2)}$.

A Política Nacional de Saúde do Idoso, a exemplo de iniciativas em outros países, prevê serviços alternativos à admissão hospitalar prolongada, dentre eles a atenção domiciliar, que visam diminuir os custos e o número de atendimentos hospitalares, bem como proporcionar humanização no atendimento sem descuidar do acompanhamento de idosos em condições de dependência prolongada.

Procurando focalizar o microcontexto hospitalar de uma instituição eleita como campo de ensino da prática profissional pelo Curso de Graduação de Enfermagem, da Universidade de Marília-SP, em particular durante a disciplina de Enfermagem Médico-Cirúrgica, foram observados episódios freqüentes de readmissões de idosos, na unidade de admissão clínica de um hospital geral e de médio porte, localizado na região sul de Marília. Esse fato foi confirmado através de estudo realizado no Hospital de Clínicas de Marília, em 1994, que pesquisou os problemas apresentados pelos idosos no momento da alta hospitalar, constatando que, dos 73 idosos pesquisados, $11 \%$ foram readmitidos num período inferior a 50 dias e também pela consulta aos dados da Divisão Regional de Saúde XIV - Marília (DIR XIV), no período de julho de 1997 a junho de $1998^{(3)}$.

A evidência das admissões repetidas dos idosos no campo do exercício profissional docente, associada a outras indagações que afloraram no grupo de trabalho assistencial, visando descobrir as razões que as geraram, quer na estrutura do serviço hospitalar, quer nas condições socioeconômicas da comunidade ou individual e familiar dos idosos, esse foi o contexto de idéias que motivou a definição do objeto a pesquisar, focalizando os eventos repetidos em idosos, nesse hospital, como subsídio para (re)conhecer a realidade do campo de ensino adotado para formação profissional do Curso de graduação de Enfermagem, em Marília-SP.

\section{OBJETIVO}

O objetivo geral desta pesquisa é caracterizar as readmissões de idosos residentes no município de Marília$\mathrm{SP}$, que apresentaram duas ou mais ocorrências num hospital geral e de médio porte, durante o período de 01/ 01/1996 a 31/12/1997, cuja fonte financiadora foi o Sistema Único de Saúde (SUS), considerando as variáveis: idade, sexo, naturalidade, estado civil, tempo de permanência da admissão, diagnósticos (principal e secundários), tipo de procedimento, tipo de saída, intervalo entre as admissões e os registros da equipe de enfermagem nos momentos da admissão e da alta.

\section{MATERIAL E MÉTODO}

Esta pesquisa tem caráter epidemiológico e descritivo. Consiste num estudo transversal e retrospectivo, realizado no período referido, num hospital geral de médio porte, do município Marília-SP, denominado "Hospital A". O município de Marília está situado na Região Centro-Oeste do Estado de São Paulo, cuja atividade econômica está voltada para pecuária e indústria 
alimentícia, além de contar com várias instituições de ensino superior.

A instituição hospitalar desta pesquisa localizase na região sul do município de Marília-SP e possui 110 leitos disponíveis para atendimento clínico e cirúrgico de diversas especialidades e maternidade.

As readmissões hospitalares podem ser classificadas em planejadas e eventuais, entendendo-se a "planejada" como necessária para a continuidade da avaliação diagnóstica ou terapêutica ${ }^{(4)}$.

Nesta investigação, o termo "readmissão" foi empregado para caracterizar os eventos de admissão hospitalar dos idosos que ocorreram duas vezes ou mais, no período em estudo de 24 meses; e "hospital geral de médio porte" representa instituição destinada a atender pessoas portadoras de doenças nas várias especialidades médicas e com capacidade de 50 a 150 leitos $^{(4-5)}$.

A pesquisa investigou a população representada pelos idosos residentes no município de Marília-SP que, no período referido, foram admitidos no Hospital "A" duas vezes ou mais.

Neste estudo foram considerados idosos indivíduos de ambos os sexos com idade igual ou superior a sessenta (60) anos, de acordo com o critério cronológico estabelecido na Política Nacional do Idoso ${ }^{(6)}$.

A primeira fonte de informações acessada foi o banco de dados do Serviço de Informações Hospitalares do Sistema Único de Saúde (SIH/SUS), de Marília, no período de 1996 e 1997, para identificar o total das admissões hospitalares da instituição em estudo, Hospital "A", e localizar as readmissões de idosos.

Na busca de definição da população de idosos a investigar, fez-se necessário selecionar todos os idosos internados no período referido e compor uma relação em ordem alfabética. Tal conduta possibilitou a identificação de homônimos e dados repetidos (número de matrícula, idade, sexo e código de endereçamento postal) a serem excluídos. Para certificar-se de que os dados e os homônimos relacionados correspondiam aos idosos que apresentaram mais de uma admissão, utilizou-se uma segunda fonte de dados disponível na Instituição, o "Livro de Registros e Admissão". Esse instrumento permitiu completar as informações, bem como ampliá-las.

Os dados permitiram gerar nova relação de idosos, com as caracterizações ampliadas e indicar os idosos que foram admitidos duas vezes ou mais.

A terceira fonte de informações utilizada foi o prontuário dos idosos, para identificar os diagnósticos secundários e registros da equipe de saúde, referentes aos momentos da admissão e alta hospitalar.

A análise das readmissões hospitalares entre idosos visa caracterizar esses eventos no período de estudo, a partir dos dados coletados nos prontuários, que constituem o primeiro banco de dados, a ser digitado e analisado através do programa (software) Epi Info 6, a partir das variáveis definidas.

A inquietação com as questões éticas da pesquisa têm gerado medidas disciplinadoras, através da constituição de comissões de ética em instituições educacionais e assistenciais. A pesquisa, portanto, só foi iniciada após consentimento livre e esclarecido da Instituição, com subscrição à Comissão de Ética da Instituição Hospitalar "A", a qual se manifestou favorável, por escrito.

\section{RESULTADOS}

No período de $1^{\circ}$ de janeiro de 1996 a 31 de dezembro de 1997, o Hospital "A", situado no município de Marília-SP, apresentou 8102 admissões, sendo 62,2\% do sexo feminino. A Tabela 1 mostra o total das admissões no período referido, segundo sexo e faixa etária, observando que o segmento etário de 60 anos ou mais representa $36,8 \%$ dos eventos.

Tabela 1 - Distribuição das admissões, segundo sexo e faixa etária, no Hospital "A”, em Marília-SP, 1996-1997

\begin{tabular}{lrrrrrrr}
\hline Período & \multicolumn{5}{c}{$1996-1997$} \\
\hline Sexo & Masculino & Feminino & \multicolumn{2}{c}{ Total } \\
Faixa etária & n.o & $\%$ & n.o & $\%$ & n.o & $\%$ \\
\hline 0-14 anos & 150 & 1,8 & 132 & 1,6 & 282 & 3,4 \\
$15-59$ anos & 1635 & 20,2 & 3194 & 39,4 & 4829 & 59,6 \\
$\begin{array}{l}\text { 60 anos ou } \\
\text { mais }\end{array}$ & 1271 & 15,7 & 1709 & 21,1 & 2980 & 36,8 \\
Ignorado & 04 & 0,05 & 07 & 0,1 & 11 & 0,2 \\
\hline \multicolumn{1}{c}{ Total } & $\mathbf{3 0 6 0}$ & $\mathbf{3 7 , 8}$ & $\mathbf{5 0 4 2}$ & $\mathbf{6 2 , 2}$ & $\mathbf{8 1 0 2}$ & $\mathbf{1 0 0}$ \\
\hline
\end{tabular}


Das 2980 admissões de idosos, $82 \%$ foram financiadas pelo SUS. O número de admissões de idosos, no Hospital "A", residentes no município de Marília-SP, totalizou 2598 , representando $87,2 \%$ do conjunto analisado (2980). Desses, foram identificados 1566 idosos em que a admissão ocorreu uma única vez e 344 idosos que foram admitidos duas ou mais vezes, representando 1032 (Tabela 2).

Tabela 2 - Número e percentual de pacientes com 60 anos ou mais, segundo as admissões geradas no Hospital "A", residentes em Marília-SP, 1996-1997

\begin{tabular}{|c|c|c|c|c|}
\hline \multirow{2}{*}{$\begin{array}{l}\text { Número de } \\
\text { vezes das } \\
\text { admissões }\end{array}$} & \multicolumn{2}{|c|}{$\begin{array}{l}\text { Número de } \\
\text { pacientes }\end{array}$} & \multicolumn{2}{|c|}{$\begin{array}{l}\text { Número de } \\
\text { admissões }\end{array}$} \\
\hline & 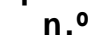 & $\%$ & n. o & $\%$ \\
\hline Uma & 1566 & 82,0 & 1566 & 60,3 \\
\hline Duas ou mais & 344 & 18,0 & 1032 & 39,7 \\
\hline Total & 1910 & 100 & 2598 & 100,0 \\
\hline
\end{tabular}

A Tabela 3 apresenta 344 idosos, residentes no município de Marília-SP, admitidos duas ou mais vezes, durante o período de estudo. O maior número dessas foi financiado pelo SUS, 92,2\%, e os demais custeados por convênios e particulares. Assim, optou-se por pesquisar as readmissões de idosos financiadas pelo Sistema Único de Saúde.

Tabela 3 - Número e percentual de pacientes com 60 anos ou mais, que foram readmitidas, segundo a fonte de financiamento, no Hospital "A", residentes em Marília-SP, 1996-1997

\begin{tabular}{lcccc}
\hline \multirow{2}{c}{$\begin{array}{c}\text { Fonte de } \\
\text { financiamento }\end{array}$} & \multicolumn{2}{c}{$\begin{array}{c}\text { Pacientes } \\
\text { n. } .\end{array}$} & $\%$ & \multicolumn{2}{c}{ Admissões } \\
& & & & $\%$ \\
\hline SUS & 310 & 90,1 & 952 & 92,2 \\
Convênio & 26 & 7,6 & 64 & 6,2 \\
Particular & 08 & 2,3 & 16 & 1,6 \\
\hline Total & $\mathbf{3 4 4}$ & $\mathbf{1 0 0}$ & $\mathbf{1 0 3 2}$ & $\mathbf{1 0 0}$ \\
\hline
\end{tabular}

Dos 344 idosos residentes em Marília-SP, que foram readmitidas no período de 24 meses, 310 foram assistidos pelo SUS e geraram 952 admissões, sendo possível localizar e coletar dados em 764 prontuários (que correspondem ao número de admissões). Nesse hospital não há modelo de prontuário único.

A análise dos dados, realizada em 764 prontuários, representou as admissões de 262 idosos, sendo 141 $(53,8 \%)$ mulheres e $121(46,2 \%)$ homens. Quanto à faixa etária, a média de idade alcançou 73,2 anos.

Outro aspecto analisado foi a freqüência das readmissões entre idosos, de ambos os sexos, conforme ilustra a Tabela 4 a seguir.
Tabela 4 - Freqüência e percentual das readmissões dos idosos, financiadas pelo SUS, segundo sexo e o número de vezes, no Hospital "A", residentes em Marília-SP, 19961997

\begin{tabular}{cccccc}
\hline $\begin{array}{c}\text { Número de } \\
\text { readmissões }\end{array}$ & Masculino & $\begin{array}{c}\text { Sexo } \\
\text { Feminino }\end{array}$ & $\begin{array}{c}\text { Total } \\
\text { Todal das } \\
\text { readmissões } \\
\text { n.o }\end{array}$ \\
\hline 1 & 77 & 88 & $\mathbf{1 6 5}$ & $\mathbf{1 6 5}$ & 32,8 \\
2 & 20 & 22 & $\mathbf{4 2}$ & $\mathbf{8 4}$ & 16,7 \\
3 & 10 & 18 & $\mathbf{2 8}$ & $\mathbf{8 4}$ & 16,7 \\
4 & 6 & 4 & $\mathbf{1 0}$ & $\mathbf{4 0}$ & 8,0 \\
5 & 2 & 4 & $\mathbf{6}$ & $\mathbf{3 0}$ & 6,0 \\
6 & 2 & 1 & $\mathbf{3}$ & $\mathbf{1 8}$ & 3,6 \\
7 & 3 & 1 & $\mathbf{4}$ & $\mathbf{2 8}$ & 5,6 \\
8 & 0 & 1 & $\mathbf{1}$ & $\mathbf{8}$ & 1,6 \\
9 & 1 & 0 & $\mathbf{1}$ & $\mathbf{9}$ & 1,8 \\
14 & 0 & 1 & $\mathbf{1}$ & $\mathbf{1 4}$ & 2,8 \\
22 & 0 & 1 & $\mathbf{1}$ & $\mathbf{2 2}$ & 4,4 \\
\hline Total & $\mathbf{1 2 1}$ & $\mathbf{1 4 1}$ & $\mathbf{2 6 2}$ & $\mathbf{5 0 2}$ & $\mathbf{1 0 0}$ \\
\hline
\end{tabular}

Dos 262 idosos estudados, destacam-se 165 $(32,8 \%)$ que foram readmitidos uma vez em dois anos, aparecendo também grande contingente de idosos que teve duas e três readmissões (33,4\%), alcançando até 22 ocorrências (4,4\%), no período.

Quanto à naturalidade dos idosos readmitidos, a maioria, 123 (46,9\%), era procedente de outros Estados brasileiros, sendo 58 (22,1\%) do próprio Estado de São Paulo e 70 (26,8\%) naturais do município de Marília e região. Dos idosos readmitidos, $75,1 \%$ eram aposentados, $62 \%$ dos homens eram casados e $58,2 \%$ das mulheres, viúvas.

A média do tempo de permanência hospitalar foi 5,71 dias, tendo duração mínima de um e máxima de 23 dias. Das 502 readmissões, 59\% tiveram alta hospitalar até o quinto dia de admissão e $95,2 \%$ saíram até o nono dia de hospitalização.

Quanto ao tipo de procedimento realizado, 96,6\% representaram o "tratamento clínico".

As principais causas das readmissões destacam as doenças do aparelho respiratório com $183(36,4 \%)$ e as doenças do aparelho circulatório com 169 (33,6\%), representando juntas $70 \%$ do total de eventos. As doenças do aparelho respiratório foram mais freqüentes para ambos os sexos e pouco mais expressiva nas mulheres.

Ocuparam o terceiro lugar as patologias do aparelho digestivo, com pequeno número em relação às anteriores, ou seja, 39 readmissões, representando $7,8 \%$ das causas, sendo $8,5 \%$ para o sexo masculino e $7,2 \%$ para o feminino, constando com maior freqüência as doenças do esôfago, estômago e duodeno.

As doenças do sistema osteomuscular e tecido 
conjuntivo apresentaram escores muito próximos para ambos os sexos, ocupando o quarto lugar dentre os diagnósticos principais e foram responsáveis por 27 readmissões, equivalendo a $5,4 \%$.

O grupo de diagnóstico representado pelos sintomas, sinais e afecções mal definidos foi responsável apenas por 20 readmissões (4\%), em que se destacaram as mulheres.

A doença pulmonar obstrutiva crônica (DPOC) foi a causa de 138 eventos (27,5\%), sendo pequena a diferença entre os sexos. As infecções respiratórias ocuparam o terceiro lugar, como causa de 33 readmissões que, somadas às anteriores, representam cerca de $1 / 3$ dos eventos por problemas respiratórios, sendo numericamente maior nas mulheres. Agrupadas as doenças dos aparelhos respiratório e circulatório representaram $2 / 3$ das readmissões. Cabe ressaltar que as doenças do aparelho circulatório se destacaram mais nos homens que nas mulheres, 36\% e 31,9\%, respectivamente, e o diagnóstico mais freqüente foi a insuficiência cardíaca com pouca diferença entre os sexos.

Destaca-se que, nos 262 idosos, 119 (45,4\%) apresentavam pelo menos mais uma doença associada ao diagnóstico principal, na primeira admissão, denominada, neste estudo, diagnóstico secundário. Das 502 readmissões de idosos analisadas, 280 (55,8\%) tinham um diagnóstico secundário e $76(15,1 \%)$ dois diagnósticos secundários. As doenças do aparelho circulatório predominaram entre as causas secundárias, destacando-se a hipertensão arterial e a insuficiência cardíaca.

Considerando que 262 idosos geraram 764 admissões, foram analisados os intervalos de tempo entre as 502 readmissões. Observou-se que 281 (55,9\%) ocorreram no período inferior a 60 dias, revelando a gravidade da condição do idoso, pois cerca de $80 \%$ aconteceram antes de 180 dias após a alta hospitalar, considerado período crítico para esses eventos.

Das readmissões estudadas, 469 (93,4\%) receberam alta com estado de saúde melhorado e 23 $(8,7 \%)$ faleceram, sendo 14 homens e 9 mulheres. Dentre os 23 idosos que foram a óbito houve pequeno intervalo entre os eventos. Nove (39\%) idosos foram readmitidos no intervalo inferior a uma semana e faleceram, enquanto 14 (61\%) retornaram à instituição em menos de um mês.

Buscando ampliar o entendimento do fenômeno das readmissões hospitalares dos 262 idosos, em estudo, foram consultados os 764 prontuários com vistas a identificar o conteúdo dos registros feitos pela enfermagem à admissão e alta hospitalar.

A análise do conteúdo dessas informações permitiu identificar as condições de dependência ou não dos idosos para a locomoção até o hospital, bem como as alterações clínicas que motivaram as admissões.

O primeiro conjunto temático sobre a "admissão" faz referência ao acompanhamento do idoso e/ou seu motivo, ilustrado com segmentos dos registros, tais como: admitido acompanhado pelo filho com dispnéia, cefaléia e fraqueza; veio deambulando, acompanhado da filha e refere dor abdominal; admitido para tratamento clínico, acompanhado pela esposa, com falta de ar. Foram encontrados 478 registros com informações semelhantes.

O segundo conjunto de registros, contendo 151 anotações, enfatiza apenas as alterações clínicas, que justificaram a admissão, sem alusão ao acompanhante ou estar só, a exemplo: chegou com tosse e cefaléia; admitido na unidade com dispnéia e tosse; chegou com dores generalizadas.

O terceiro segmento temático destaca as condições de dependência para locomoção, além da presença do acompanhante e alterações clínicas, compostas por 135 registros, ilustrados com trechos do tipo: veio acompanhado pelo filho em cadeira de rodas, dispnéico, edema de membros inferiores e fraqueza; admitido na unidade em cadeira de rodas com tontura.

Predominaram os registros sobre as condições clínicas. Dos 764 prontuários analisados, 246 (32\%) tinham a queixa de dispnéia. Além dessa manifestação respiratória, outras foram identificadas, por ordem decrescente, a saber: tosse, fraqueza, dor no tórax, dor abdominal, cefaléia, dor lombar, dor generalizada, mal estado geral, edema de membros inferiores, hipertensão, diarréia, vômito, tontura e falta de ar, entre outras.

A análise dos registros possibilitou detectar que os funcionários seguem um padrão de informações sobre as condições do paciente à admissão, acrescidas da notificação de três sinais vitais (pressão sangüínea arterial, pulso e temperatura corporal), sem quantificar a freqüência respiratória, apesar da dispnéia ter sido o sintoma mais referido e, no hospital em estudo, as doenças responsáveis por $1 / 3$ das admissões foram do aparelho respiratório.

Retomando os prontuários para identificar o conteúdo dos registros no momento da "alta" hospitalar, observou-se resumidas informações sobre as condições do idoso e conduta médica. Quatrocentos e dois 
prontuários (402) fizeram alusão ao acompanhante do idoso e à entrega da receita médica, conforme os exemplos: saiu acompanhado pela filha levando a receita; saiu de alta acompanhado pela esposa com a receita médica; saiu de alta com o neto levando receita.

Foram encontrados 65 (8,5\%) prontuários que não havia nenhum registro de enfermagem em relação à saída do idoso da unidade, apenas constava a assinatura do médico responsável pela autorização da alta melhorada. Somente $18(2,3 \%)$ faziam menção às orientações de enfermagem referentes à receita médica, dieta e atividade física, sendo a grande maioria (17) feita por acadêmicos do curso de graduação em enfermagem e um apenas da enfermeira responsável pela unidade.

Referências aos problemas sociais e familiares só foram identificadas em sete prontuários que relatavam situações em que a família demorou mais de 24 horas para buscar seu idoso em condição de alta hospitalar. $\mathrm{O}$ período de tempo que a família levou para retirar seu idoso variou entre um e sete dias, conforme o confronto entre as datas da saída e da decisão médica pela alta.

Em apenas cinco prontuários foram anotados os estados de dependência total ou parcial dos idosos ao saírem de alta do hospital, sendo que, em três, também foi registrada presença de sonda nasogástrica para oferta de alimentos no domicílio.

Com base na análise do conteúdo dos registros escritos nos prontuários, à admissão e à alta, foi possível reconhecer a rotina da prática de enfermagem na instituição em estudo, que revela apenas alguns aspectos da situação dos idosos à recepção e à saída, centradas na descrição das condições clínicas que motivaram a admissão, não fazendo referência ao tipo de intervenção a que o idoso foi submetido. Nos registros de saída constava apenas a presença ou não de acompanhante e a entrega da receita médica. Tais constatações alertam para a necessidade de se rever o processo de trabalho e gerenciamento da assistência de enfermagem, incluindo a avaliação do paciente, indicando o diagnóstico que direcione o planejamento e a implementação das ações de enfermagem, no decorrer do período entre a admissão e a alta hospitalar, prevendo a continuidade do cuidado no domicílio.

\section{DISCUSSÃO DOS DADOS}

O presente estudo identificou 262 idosos que foram responsáveis por 764 admissões, retratando 502 episódios no hospital. O grupo foi composto por $54 \%$ de idosos do sexo feminino. Esses dados não são diferentes dos estudos realizados em Ribeirão Preto-SP, em que a participação do sexo feminino também foi relativamente maior $^{(7-8)}$

Apesar do período estudado ser maior que o adotado por outros autores, o índice de readmissões de 18\% não difere de outras pesquisas. Em Aberdeen (EUA), o intervalo de tempo definido para ser considerada readmissão foi menor ou igual a 28 dias, num estudo com idosos hospitalizados por período de seis meses, em que foram evidenciados 109 eventos entre 713 altas, gerando $15,3 \%$ readmissões ${ }^{(9)}$.

Na Suíça, pesquisa desenvolvida com 5828 idosos que receberam alta hospitalar, num período de doze meses, foram detectadas 1796 readmissões, representando $30,8 \%$ de ocorrências ${ }^{(4)}$.

No presente estudo, a média de permanência hospitalar ficou em 5,71 dias, enquanto a média para o grupo etário de 60 anos ou mais, no Brasil, foi 7,1 dias em admissões financiadas pelo SUS, referentes ao ano de 1995. As baixas médias obtidas pela população idosa podem ser explicadas pelo sistema de pagamento adotado pelo governo em relação aos hospitais conveniados, que adota como critério apenas uma enfermidade como motivo da admissão hospitalar, independente das enfermidades associadas que o sujeito possa apresentar. Talvez, essa seja uma pista para buscar explicações sobre a ocorrência das altas precoces para muitos idosos, que desencadearam as freqüentes readmissões ${ }^{(10)}$.

O idoso é mais vulnerável às infecções respiratórias devido às diversas mudanças estruturais e funcionais que se instalam com a senescência; o processo de envelhecimento afeta também progressivamente o sistema cardiovascular.

Dentre as principais causas de admissões no Hospital "A", destacaram-se as doenças do aparelho respiratório e circulatório, acarretando a readmissão de $55 \%$ dos idosos num intervalo de tempo inferior a 60 dias; entre aqueles que havia dois diagnósticos secundários, 0 intervalo diminuiu de 01 a 29 dias.

No município de São Carlos-SP, o primeiro lugar coube às doenças do aparelho circulatório com 31,7\%, ficando os sintomas, sinais e afecções mal definidas em segundo, com $15,2 \%$ e aquelas do aparelho respiratório 
com 13,6\%, em terceiro plano; as doenças do aparelho digestivo em quarto, com $9,2 \%$ e do aparelho geniturinário com $5,6 \%$, todas responsáveis pelas readmissões de idosos, nos hospitais do município no período de $1996^{(1)}$.

Estudo realizado em Genebra, Suíça, entre julho de 1995 a junho de 1996, com 730 adultos que foram readmitidos num período de 31 dias, indicou vários fatores de risco para as ocorrências, dentre eles a idade (indivíduos com mais de 74 anos), a longa permanência hospitalar na primeira admissão e o número de morbidades associadas, principalmente as doenças coronarianas, falência cardíaca, arritmias cardíacas, embolia pulmonar, doenças cerebrovasculares, neoplasmas, DPOC e pneumonias; apontam as doenças circulatórias em maior número com $27,9 \%^{(4)}$.

A análise do conteúdo dos registros feitos pela enfermagem, nos prontuários dos idosos, permite caracterizar o modelo de atenção hospitalar. Destaca-se o seu caráter mecanicista, fragmentado e direcionado para o desempenho de funções assistenciais de enfermagem que cumprem as prescrições médicas.

Estudos sobre anotações de enfermagem afirmam que a precariedade de informações retrata a forma taylorizada da assistência de enfermagem por enfatizarem apenas o descrito relativo às terapêuticas clínico-cirúrgicas

\section{REFERÊNCIAS BIBLIOGRÁFICAS}

1. Caselli MP A morbidade hospitalar do idoso no município de São Carlos/SP em 1996. [dissertação] São Paulo (SP): Faculdade de Saúde Pública/USP; 1998.

2. Silvestre JA Programa de Saúde do Idoso. Saúde do Idoso. Programas e Projetos. [online] 1999 [citado em 10/10/1999]. Disponível em:URL: http://www.saude.gov.br

3. Marin MJS Levantamento de problemas dos pacientes idosos no momento da alta hospitalar. [dissertação]. Ribeirão Preto (SP): Escola de Enfermagem de Ribeirão Preto/USP; 1995.

4. Kossovsky MP, Perneger TV, Sarasin FP, Bolla F. Comparasion between planned and unplanned readmissions to a Department of Internal Medicine. J Clin Epidemiol 1999 February; 52(2):151-6.

5. Purita F. Práticas de medicina hospitalar. Rio de Janeiro: Cultura Médica; 1992.

6. Lei n. 8.842. Dispõe sobre a Política Nacional do Idoso e cria o Conselho Nacional do Idoso. 1994. 05 de janeiro, Seção I, p.152-6.

7. Rodrigues RAP. O Paciente geriátrico cirúrgico em hospitais gerais de Ribeirão Preto, 1983. [dissertação]. Ribeirão Preto (SP): Escola de Enfermagem de Ribeirão Preto/USP; 1987. em detrimento das anotações referentes às condições fisiológicas e às intervenções de enfermagem. O registro adequado das anotações de enfermagem deve ser entendido como atitude de cuidar com qualidade e eficiência junto ao ser humano ${ }^{(11-12)}$.

\section{CONSIDERAÇÕES FINAIS}

Algumas reflexões se fazem necessárias, a saber: retomar a concepção humanista para focalizar o processo saúde-doença na pessoa idosa, de modo a incorporar os pressupostos do cuidado compreensivo e ampliar as relações entre sujeitos e estruturas sociais envolvidas nas ações promocionais, mantenedoras e recuperadoras da saúde.

E, ainda, cabe relevar um dos caminhos a trilhar, seja resgatando dinâmicas de discussão em grupo sobre algumas dessas questões, junto aos diversos agentes de saúde nos contextos dos serviços. Refletir profundamente sobre as responsabilidades das instituições diante das demandas reais da sociedade e dos profissionais nas diversas áreas de assistência às pessoas idosas, famílias e comunidade, de modo a introduzir modificações no modelo de atenção e equipamentos sociais de apoio.

8. Germano J Neto O Paciente geriátrico no hospital, Ribeirão Preto/SP, 1988.[dissertação] Ribeirão Preto (SP): Faculdade de Medicina de Ribeirão Preto/USP; 1991.

9. Gautam P, Macduff C, Brown I, Squair J. Unplanned readmissions of elderly patients. Health Bull 1996 November; 54(6):449-57.

10. Silvestre JA, Kalache A, Ramos LR, Veras RP. O envelhecimento populacional brasileiro e o setor saúde. Arq Geriatr Gerontol 1996 Setembro; 1:81-9.

11. Pádua A. Análise das anotações de enfermagem: uma proposta de sistematização da assistência de enfermagem. [dissertação] São Paulo (SP): Escola Paulista de Medicina/ UNIFESP; 2000.

12. Canello BL, Muntsch S. Projeto de implantação de enfermagem em unidade de terapia intensiva pediátrica. Rev Bras Enfermagem 1998 Abril-Junho; 51(2):321-36. 